\title{
Identification of microRNAs differentially expressed between lung squamous cell carcinoma and lung adenocarcinoma
}

\author{
JUNKO HAMAMOTO $^{1}{ }$, KENZO SOEJIMA ${ }^{1}$, SATOSHI YODA ${ }^{1}$, KATSUHIKO NAOKI ${ }^{1}$, SOHEI NAKAYAMA ${ }^{1}$, \\ RYOSUKE SATOMI $^{1}$, HIDEKI TERAI ${ }^{1}$, SHINNOSUKE IKEMURA ${ }^{1}$, TAKASHI SATO ${ }^{1}$, HIROYUKI YASUDA ${ }^{1}$, \\ YUICHIRO HAYASHI $^{2}$, MICHIIE SAKAMOTO ${ }^{2}$, TORU TAKEBAYASHI ${ }^{3}$ and TOMOKO BETSUYAKU ${ }^{1}$
}

Departments of ${ }^{1}$ Pulmonary Medicine, ${ }^{2}$ Pathology, and ${ }^{3}$ Preventive Medicine and Public Health, School of Medicine, Keio University, Tokyo 160-8582, Japan

Received February 7, 2013; Accepted June 3, 2013

DOI: $10.3892 / \mathrm{mmr} .2013 .1517$

\begin{abstract}
Recent advances in the treatment of non-small cell lung cancer (NSCLC) with new agents require accurate histological subtyping at diagnosis to avoid the higher risk of an adverse response and to obtain the maximum therapeutic response. However, interobserver variability, tumor heterogeneity and the degree of differentiation may affect the decision concerning a pathological diagnosis of NSCLC. Therefore, the aim of this study was to identify specific microRNAs (miRNAs) as standardized biomarkers with high sensitivity and specificity in order to distinguish between squamous cell carcinoma (SCC) and adenocarcinoma (AC). Quantitative polymerase chain reaction (qPCR)-based miRNA array analysis was performed to identify microRNAs differentially expressed between SCC and AC using 86 resected NSCLC samples in addition to adjacent normal tissues. The results were confirmed by independent qRT-PCR assays with the same test samples and 88 additional validation samples, and from this we evaluated the usefulness of the identified miRNAs as biomarkers to distinguish between SCC and AC. Three miRNAs (hsa-miR-196b, hsa-miR-205 and hsa-miR-375) were identified. Discriminant analysis combining the three miRNAs appeared to distinguish SCC from AC accurately in the test and validation samples, demonstrating a sensitivity and specificity of 76 and $80 \%$, and 85 and $83 \%$, respectively. hsa-miR-196b, hsa-miR-205 and hsa-miR375 were identified as biomarkers capable of distinguishing between lung SCC and lung AC. These newly identified miRNAs may prove to be highly valuable molecular markers for the classification of NSCLC histological subtypes and may contribute to the pathogenesis of each subtype of NSCLC.
\end{abstract}

Correspondence to: Dr Kenzo Soejima, Department of Pulmonary Medicine, School of Medicine, Keio University, 35 Shinanomachi, Shinjuku-ku, Tokyo 160-8582, Japan

E-mail: ksoejima@cpnet.med.keio.ac.jp

Key words: lung cancer, squamous cell carcinoma, adenocarcinoma, microRNA, biomarker

\section{Introduction}

Lung cancer is the leading cause of cancer mortalities worldwide. The main type of lung cancer is non-small cell lung cancer (NSCLC), which may be divided into three histologic subtypes: squamous-cell carcinoma (SCC), adenocarcinoma (AC), and large-cell lung carcinoma (LCC) (1). Data from recent clinical trials suggest a significant interaction between treatment efficacy and tumor histology (2-7). Analysis of a phase III study with pemetrexed demonstrated that survival was superior in non-squamous NSCLCs compared to $\operatorname{SCC}(2,3)$. This finding may be explained by the fact that thymidylate synthase, one of the main targets of pemetrexed, was found to be differentially expressed among the histotypes of lung cancer, with a lower expression in $\mathrm{AC}$ and a higher expression in SCC and small-cell lung cancer $(4,5)$. Although the most important prediction factor of an epidermal growth factor receptor-tyrosine kinase inhibitor (EGFR-TKI) has been reported to be the EGFR mutation status rather than the histology, the histology of $\mathrm{AC}$ remains an important clinical predicting factor for EGFR-TKI, particularly gefitinib, in the case that the mutation status is not obtained $(6,7)$. Bevacizumab, a targeted therapy agent that inhibits tumor angiogenesis, has only been registered for the treatment of non-SCC in the USA, Europe and other countries due to serious hemorrhagic complications in SCC $(3,8)$. Therefore, histological characterization is the milestone for tailored therapy, which is important in order to maximize the benefits of novel drugs and to avoid hazardous side effects.

Immunohistochemical markers including TTF-1, p63, cytokeratin 7 and high-molecular-weight cytokeratins have been identified for subtyping AC and SCC. However, the sensitivity and specificity of these diagnostic markers requires further definition (5). Interobserver variability, tumor heterogeneity and the degree of differentiation may also contribute to variability in the histological subtyping of NSCLC. microRNAs (miRNAs) are an abundant class of small nonprotein coding RNAs that function as negative gene regulators. miRNAs regulate diverse biological processes, and evidence has shown that miRNA mutations or the aberrant expression of miRNA are correlated with various types of human cancer (9). Several miRNAs have been shown to repress the expression of 
Table I. Clinical characteristics of 86 test samples obtained from NSCLC patients.

\begin{tabular}{|c|c|c|c|c|c|}
\hline Characteristics & $\mathrm{AC}$ & $\mathrm{SCC}$ & $\mathrm{LCC}$ & LCNEC & Total \\
\hline Number of cases & 54 & 25 & 4 & 3 & 86 \\
\hline Median age (range) & $65(36-83)$ & $72(56-83)$ & $59(51-79)$ & $76(62-80)$ & $68(36-83)$ \\
\hline \multicolumn{6}{|l|}{ Gender } \\
\hline Male & 27 & 22 & 4 & 3 & 56 \\
\hline Female & 27 & 3 & 0 & 0 & 30 \\
\hline \multicolumn{6}{|l|}{ Smoking } \\
\hline Ever & 29 & 22 & 3 & 2 & 57 \\
\hline Never & 23 & 2 & 1 & 1 & 26 \\
\hline NA & 2 & 1 & 0 & 0 & 3 \\
\hline \multicolumn{6}{|l|}{ Stage } \\
\hline I & 40 & 19 & 3 & 2 & 64 \\
\hline II & 5 & 3 & 1 & 1 & 10 \\
\hline I & 5 & 3 & 0 & 0 & 8 \\
\hline IV & 1 & 0 & 0 & 0 & 1 \\
\hline NA & 3 & 0 & 0 & 0 & 3 \\
\hline
\end{tabular}

AC; adenocarcinoma, SCC; squamous cell carcinoma, LCC; large cell carcinoma, LCNEC; large cell neuroendocrine carcinoma, NA; data not available.

important cancer-related genes and have proven to be useful in the diagnosis and treatment of cancer $(10,11)$.

We hypothesized that certain miRNA signatures may provide a highly sensitive and specific diagnostic test for distinguishing between SCC and AC. An initial screening of miRNA was performed in 86 test samples from NSCLC patients using a quantitative polymerase chain reaction (qPCR)-based miRNA array analysis and three miRNAs whose expression levels varied significantly between SCC and $\mathrm{AC}$ were identified. Algorithms combined with the expression levels of these three miRNAs accurately divided SCC and AC. The diagnostic assay developed was validated with an additional set of 88 clinical NSCLC samples. These newly identified miRNAs may prove to be highly attractive molecular markers for the classification of NSCLC histological subtypes, and may contribute to the pathogenesis of each subtype of NSCLC.

\section{Materials and methods}

Patients and histologic diagnosis. Anonymous surgically resected NSCLC samples obtained at the Keio University Hospital were obtained as indicated in Table I. Institution review and ethics approval was obtained for all the samples in accordance with the institutional review board of Keio University (Institutional Review Board \#16-90-1). Tumor tissues were intraoperatively dissected with the surrounding normal tissues, and paired normal lung tissues were obtained from the same patients at an adjacent area from their tumors. Serial cryostat sections from the specimens were stained with $\mathrm{HE}$ for the histological diagnosis based on the most recent WHO classification lung tumors $(12,13)$. HE-stained slides of all the cases were reviewed by Y.H. In case of any discrepancy, the slide was also reviewed by M.S. and the original pathologist who signed out the case, in order to achieve a consensus.

RNA extraction. Total RNA containing small RNA was extracted from the tumor and adjacent normal tissues obtained during surgical resection for a carcinoma located elsewhere in the lung. Frozen tissue fragments were placed in TRIzol ${ }^{\circledR}$ reagent (Invitrogen, Carlsbad, CA, USA) and homogenized with a Polytron homogenizer (Kinematica AG, Lucerne, Switzerland), followed by RNA extraction according to the manufacturer's instructions.

miRNAarray. The Megaplex RT(AppliedBiosystems, Carlsbad, CA, USA) was used to reverse transcribe 377 miRNAs using $50 \mathrm{ng}$ total RNA from each sample. The expression levels of 377 miRNAs that included control U6 snRNA were measured using TaqMan microRNA array human A v 2.0 (Applied Biosystems) according to the manufacturer's instructions.

$q R T-P C R$. The relative levels of miR-196b, miR-205 and miR-375 were determined by stem loop real-time qPCR using gene-specific primers according to the TaqMan microRNA assay instructions. U6 snRNA was selected as the normalizer, as this miRNA demonstrated minimal variation in expression among ACs and SCCs (data not shown). Each miRNA was individually amplified in triplicate. The relative levels of individual miRNAs, with reference to U6, were calculated using the $\triangle \mathrm{CT}$ method.

Data analysis and statistics. MATLAB software (MathWorks Inc., Natick, MA, USA) was used for the statistical analyses of the array data. The tumor expression level of each miRNA was calculated as the decadic logarithm of the average of all 
the normal samples. The difference in the decadic logarithm for each miRNA between SCC and AC was calculated using the t-test with MATLAB software, followed by extracting five miRNAs using a Pearson's correlation of $\mathrm{P}<0.001$. As a result, miR-196b, miR-205 and miR-375 were selected as the $\log _{10} \mathrm{AC}-\log _{10} \mathrm{SCC}$ absolute values (AC/SCC ratio) of the miRNAs, with a value of $>0.6$.

Unpaired t-test, receiver-operator characteristic (ROC) curve, correlation coefficient and discriminant formula were obtained using IBM SPSS statistics software (ver. 17.0, SPSS Inc., Chicago, IL, USA). The average Ct values of the triplicates $\left(\mathrm{AvgCt}_{\text {miR 196b }}, \mathrm{AvgCt}_{\mathrm{miR} 205}, \mathrm{AvgCt}_{\mathrm{miR} 375}, \mathrm{AvgCt}_{\mathrm{U6}}\right)$ detected by qRT-PCR were calculated (outliers differing by a $\mathrm{SD}>1$ were excluded). The sample score was obtained using the formula: Score $=\mathrm{AvgCt}_{\mathrm{miR}}-\mathrm{AvgCt}_{\mathrm{U} 6}$. The difference in the sample score for each miRNA between SCC and AC was calculated using the Mann-Whitney test as the distribution of miR-205 scores in the SCC group and miR-375 scores in the $\mathrm{AC}$ group were not assumed to be normal. ROC curves were constructed and the area under the ROC curve (AUC) was calculated with SPSS software using the score for each miRNA.

The sensitivity (\%) for each miRNA was calculated by dividing the sample number of each subtype, determined either from the cut-off value or the discriminant function by the number determined histologically. Cut-off values dividing the SCC and AC groups were selected to reveal the maximum value of the Youden index (sensitivity + specificity - 1), calculated from the ROC curves. Coefficients and constants of discriminant function were developed from discriminant analysis with SPSS software. If the z-value was positive, the sample was judged to be AC and if the z-value was negative, the sample was judged to be SCC. The discriminant formulae for the miRNAs are as follows: $Z_{\text {miR } 1966}=0.516 \times \mathrm{Ct}_{\mathrm{miR} 196 \mathrm{~b}}-5.956$, $\mathrm{Z}_{\text {miR } 205}=0.393 \times \mathrm{Ct}_{\text {miR205 }}-2.169, \mathrm{Z}_{\text {miR } 375}=-0.446 \times \mathrm{Ct}_{\text {miR375 }}+2.501$, $\mathrm{Z}_{\text {miR-196b and miR-205 }}=0.269 \times \mathrm{Ct}_{\text {miR } 196 \mathrm{~b}}+0.26 \times \mathrm{Ct}_{\mathrm{miR} 205}-4.541$, $\mathrm{Z}_{\text {miR-196b and miR-375 }}=0.277 \times \mathrm{Ct}_{\mathrm{miR} 196 \mathrm{~b}}-0.314 \times \mathrm{Ct}_{\mathrm{miR} 375}-1.422$, $\mathrm{Z}_{\text {miR-205 and miR-375 }}=0.216 \times \mathrm{Ct}_{\text {miR205 }}-0.282 \times \mathrm{Ct}_{\text {miR375 }}+0.407$, $\mathrm{Z}_{\text {combination }}=0.201 \times \mathrm{Xt}_{\text {miR } 1966}+0.144 \times \mathrm{xt}_{\text {miR205 }}-0.243 \mathrm{xCt}_{\mathrm{miR} 37^{-}}-1.736$.

\section{Results}

Identification of miRNAs differentially expressed between $A C$ and SCC. We initially performed miRNA profiling of 86 Japanese lung cancer samples that included 54 lung ACs, 25 SCCs, 4 LCCs and 3 large cell neuroendocrine carcinomas (LCNECs) using miRNA arrays (Table I). We compared the tumor miRNA expression levels relative to those in adjacent normal tissues in SCC and those in AC, and identified five differentially expressed miRNAs between the SCC and AC tumors (Fig. 1A, P<0.001). Of the five miRNAs, miR-196b, miR-205 and miR-375 exhibited significant differential expression between SCC and AC (Fig. 1B). The difference in $\log _{10} \mathrm{SCC}$ and $\log _{10} \mathrm{AC}$ was $-0.86,-1.12$ and 0.81 for miR-196b, miR-205 and miR-375, respectively. While miR-196b and miR-205 were overexpressed in SCC, miR-375 was overexpressed in AC.

Expression levels of $m i R-196 b, m i R-205$ and $m i R-375$. To confirm the array results, the expression levels of the
A

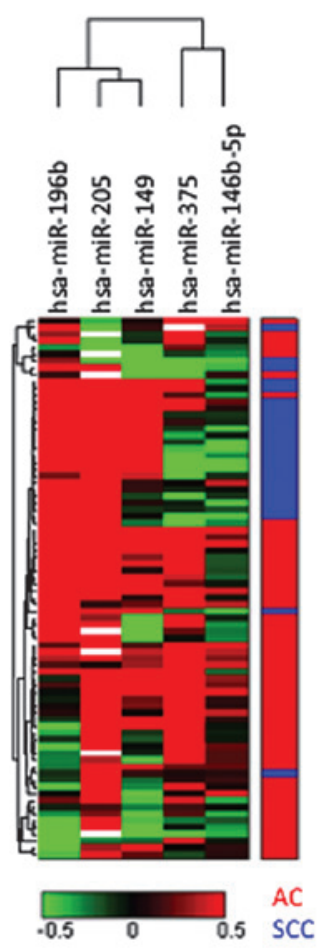

B

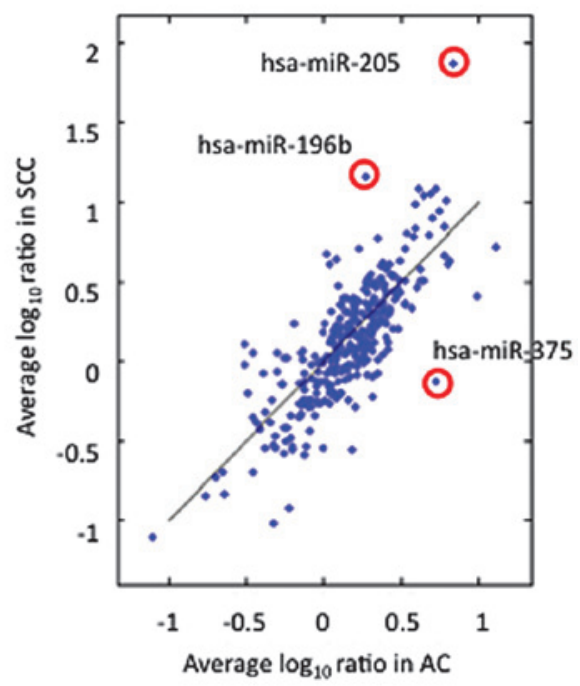

Figure 1. Identification of differentially expressed microRNAs (miRNAs) between adenocarcinoma (AC) and squamous cell carcinoma (SCC). (A) Hierarchical clustering of five miRNAs with significantly different expressions. The tumor expression level of each miRNA was calculated as the decadic logarithm of the average of all the normal samples. The difference in the decadic logarithm for each miRNA between SCC and AC was calculated by a t-test using MATLAB software (five microRNAs demonstrating a Pearson's correlation of $\mathrm{P}<0.001$ were extracted). Rows represent individual tissue samples, blue indicates SCC and red indicates AC. Columns represent individual miRNAs. The scale represents the intensity of miRNA expression ( $\log _{10}$ scale ranges between -0.5 and 0.5 ). (B) miRNAs differentially expressed in ACs vs. SCCs. The X-axis shows average decadic logarithm for each miRNA expression in AC, while the Y-axis shows average decadic logarithm for each miRNA expression in SCC. miR-196b, miR-205 and miR-375 were selected as the $\log _{10} \mathrm{AC}-\log _{10} \mathrm{SCC}$ absolute values ( $\mathrm{AC} / \mathrm{SCC}$ ratio) of those miRNAs with a value of $>0.6$.

three miRNAs and the U6 snRNA of 86 samples were measured in triplicate by qRT-PCR, independently from the array. The expression patterns of these miRNAs in LCCs were similar to those in ACs, while their expression patterns 
Table II. Sensitivity (\%) by cut-off values calculated from ROC curves.

\begin{tabular}{lccc}
\hline Variables & miR-196b & miR-205 & miR-375 \\
\hline Cut-off value & 10.15 & 2.55 & 6.65 \\
AC & $88 \%$ & $96 \%$ & $92 \%$ \\
SCC & $64 \%$ & $68 \%$ & $72 \%$ \\
\hline
\end{tabular}

Cut-off values with which to divide the patients into the squamous cell carcinoma (SCC) group and the adenocarcinoma (AC) group were determined from the scores that yielded the maximum value of the Youden index (sensitivity + specificity - 1) calculated from the receiver-operator characteristic (ROC) curves. Sensitivity (\%) for each miRNA was calculated by dividing the sample number of each subtype determined by these cut-off values.

A

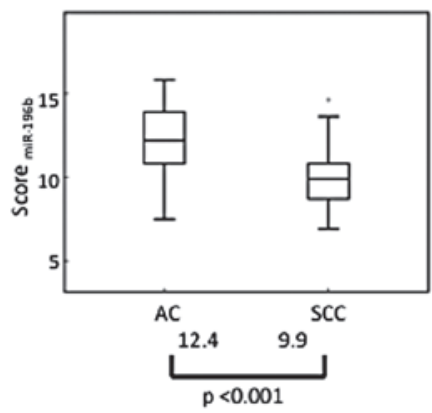

B

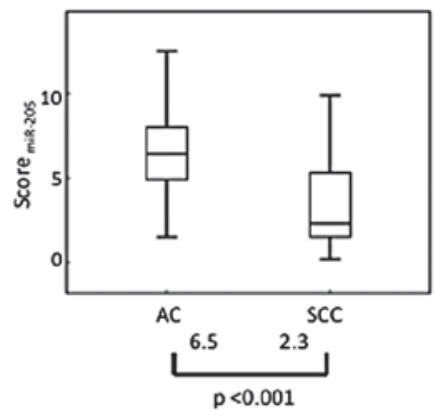

C

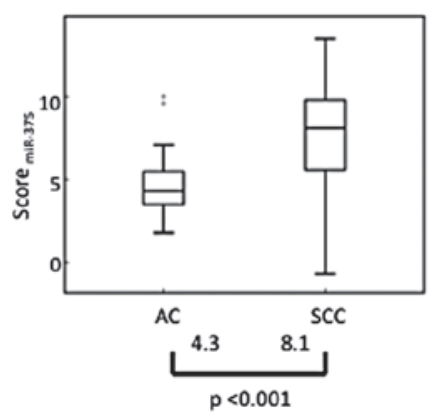

Figure 2. Difference in each miRNA score between squamous-cell carcinoma (SCC) and adenocarcinoma (AC). Median scores of the AC (left column) or SCC (right column) group are represented in the graphs [(A) miR-196b, (B) miR-205 and (C) miR-375]. The Y-axis shows a sample score congruent to $\mathrm{AvgCt}_{\mathrm{miR}}-\mathrm{AvgCt}_{\mathrm{U} 6}$. P-values were calculated by a non-parametric Mann-Whitney test with SPSS software ver. 17.0 and a statistically significant difference was observed between $\mathrm{AC}$ and $\mathrm{SCC}(\mathrm{P}<0.001)$.

in LCNECs were similar to those in SCCs (data not shown). The median values of each miRNA score varied significantly between the SCC and AC groups $(\mathrm{P}<0.001)$ (Fig. 2).
$\mathbf{A}$

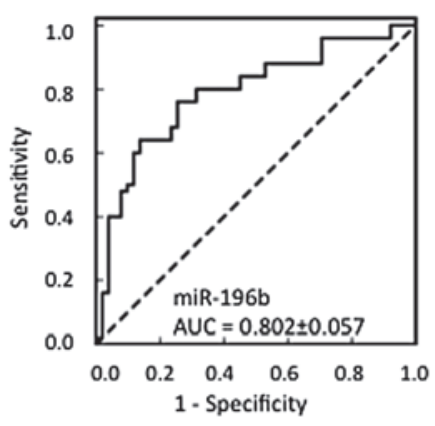

B

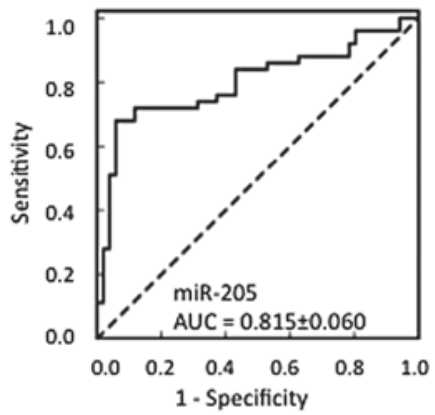

C

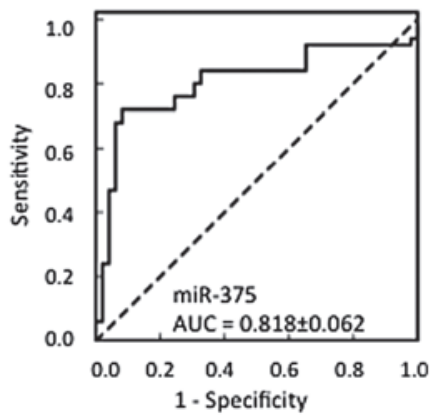

Figure 3. Receiver-operator characteristic (ROC) analysis. ROC curves of the three miRNAs of 54 adenocarcinomas (ACs) and 25 squamous cell carcinomas (SCCs). The area under the curve (AUC) was calculated using SPSS software ver. 17.0 using the score for each miRNA [(A) miR-196b, (B) miR-205 and (C) miR-375], with the sample scores of the SCC as the state variable. $\mathrm{X}$-axis shows 1 -specificity; Y-axis shows sensitivity. The dotted lines act as references.

Optimization of miRNA markers to distinguish SCC from $A C$. The AUC of the three miRNAs was $>0.8$, indicating that a diagnosis by these markers would provide moderate accuracy even as a single biomarker (Fig. 3). The cut-off value for each miRNA and the sensitivity from each cut-off value used to distinguish SCC and AC are listed in Table II. While the three miRNAs demonstrate moderate correlation with each other, 0.558 for miR-196b and $-205,-0.440$ for miR-196b and -375 and -0.568 for miR-205 and -375 , we introduced discriminant analysis by calculating the $\mathrm{z}$-value using scores of not only single miRNA but two or three miRNAs (Table III).

Validation. These classification methods with novel identified miRNA markers were validated using an independent set of 88 NSCLC samples that included 44 SCCs and 44 ACs. The expression level was measured and the sample score was calculated using the established method. The sensitivity of identifying SCC by all three miRNAs was $85 \%$ and the specificity was $83 \%$ (Table III). 
Table III. Sensitivity (\%) by discriminant analysis.

miRNAs

\begin{tabular}{|c|c|c|c|c|c|c|c|}
\hline Samples & $196 \mathrm{~b}$ & 205 & 375 & $196 b$ and 205 & $196 b$ and 375 & 205 and 375 & $196 b, 205$ a \\
\hline \multicolumn{8}{|l|}{ Test } \\
\hline $\mathrm{AC}$ & 63 & 63 & 76 & 69 & 71 & 80 & 80 \\
\hline SCC & 80 & 76 & 72 & 80 & 76 & 76 & 76 \\
\hline \multicolumn{8}{|l|}{ Validation } \\
\hline $\mathrm{AC}$ & 63 & 62 & 89 & 58 & 75 & 78 & 83 \\
\hline SCC & 88 & 86 & 78 & 91 & 80 & 85 & 85 \\
\hline
\end{tabular}

Sensitivity $(\%)$ for each miRNA was calculated by dividing the sample number of each subtype determined using discriminant function by that determined histologically. Coefficients and constants were developed from discriminant analysis with SPSS software ver. 17.0. If the z-value was positive, the sample was judged to be adenocarcinoma (AC) and if the $\mathrm{z}$ value was negative, the sample was judged to be squamous cell carcinoma (SCC). Discriminant formulae for the miRNAs are indicated in Materials and methods.

\section{Discussion}

In this study, we demonstrated that three miRNAs, hsa-miR-196b, hsa-miR-205 and hsa-miR-375, may be used for the classification of AC and SCC of the lung. miR-196b is a novel distinguishing marker of $\mathrm{AC}$ and SCC that we identified, while miR-205 and miR-375 have already been reported to be candidates of specific markers for lung SCC (14-16). We observed that a combination of these three markers was more accurate than a single marker for distinguishing SCC from AC.

Initially, we attempted to predict the histology using cut-off values calculated from the ROC curves. The AUC of the three miRNAs was $>0.8$, indicating that a diagnosis by these markers would provide moderate accuracy even with a single biomarker (Fig. 3). The sensitivity for predicting AC was high (range, 88-96\%), compared with the sensitivity of predicting SCC (range, 64-72\%). The specificity of predicting AC was relatively low, (range, $64-72 \%$ ) for each of the three miRNAs using this classification method. However, it is important to accurately diagnose SCC in order to avoid adverse events and ineffective drug administration since in the clinic there are more agents that are safe and effective for non-SCC cases only. As the correlation between the three markers was low, we believed that a higher sensitivity would be obtained by combining the data for the three markers. We calculated the prediction sensitivities by discriminant function analysis for each miRNA. In contrast to the result using cut-off values, we observed a higher sensitivity for predicting SCC and a higher specificity for predicting AC. The results also suggested that subtyping by two or three miRNAs is more appropriate for diagnosis, due to the increased sensitivity for SCC and AC rather than by subtyping for a single miRNA. The result was confirmed with validation samples and only the combination of all three samples demonstrated $>80 \%$ sensitivity for the two histological types. It is thought that the z-value calculated by discriminant analysis would have more generality than the cut-off value determined by $\mathrm{Ct}$ score as the $\mathrm{z}$-value is normalized, while the $\mathrm{Ct}$ score itself is a relative value and may differ in each instituion or equipment used. Therefore, classification by discriminant analyses using three miRNAs is

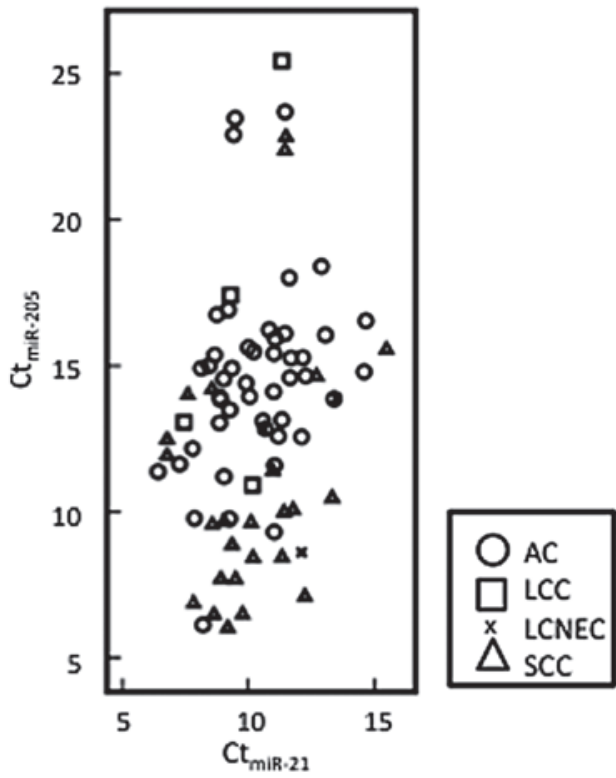

Figure 4. Expression levels of hsa-miR-205 and hsa-miR-21. The X-axis shows $\mathrm{AvgCt}_{\mathrm{miR} 21}$, and the Y-axis shows $\mathrm{AvgCt}_{\mathrm{miR} 205}$. SCC ( $\mathrm{n}=25$; triangle $)$ and LCNEC ( $\mathrm{n}=3$; cross) have high levels (low $\mathrm{Ct}$ values) of hsa-miR-205 compared with AC ( $n=54$, circle) and LCC $(n=4$, square). AC; adenocarcinoma, LCC; large cell carcinoma, LCNEC; large cell neuroendocrine carcinoma, SCC; squamous cell carcinoma.

recommended for the future classification of SCC and AC. As the size of the coefficient is proportional to the magnitude of the impact of each miRNA, miR-375 is thought to be the most effective factor among the three miRNAs for discrimination in all combinations. The significance of a combination might be increased by opposite-type markers, such as miR-375, which is overexpressed in AC, and miR-196b or miR-205, which are overexpressed in SCC.

Lebanony et al and Bishop et al reported that miR-205 alone sufficiently distinguished SCC from non-SCC and they obtained a straight line between SCC and non-SCC when they plotted the expression levels of normalized miR-205 and miR-21 $(14,16)$. We applied the same approach to our test 
samples, however, we did not obtain a clear line to distinguish histology types as shown in Fig. 4. Although we also observed that SCC expressed higher levels of miR-205 compared with $\mathrm{AC}$, the expression pattern of miR-205 was more complex. Their formula, using U6 and miR-21, revealed only $72 \%$ sensitivity in the identification of SCC in our experiments, although 96 or $100 \%$ was previously reported $(14,16)$. Although it is difficult to explain this discrepancy, it may be due to ethnic differences or heterogeneity of the tissue samples.

Overexpression of miR-196b has been observed in colon cancer or in leukemia associated with the mixed-lineage leukemia (MLL) gene, leading to increased proliferative capacity and survival, in addition to a partial block in the differentiation specifically in bone marrow progenitor cells $(17,18)$. miR-196a, a member of the miR-196b family, was reported to increase the development of lung metastases in mice following tail vein injection (19). High levels of miR-196a were found to activate the AKT signaling pathway as indicated by the increased phosphorylation of AKT and promoted cancer cell detachment, migration and invasion but did not have an impact on proliferation or apoptosis (19).

It has been verified that miR-205 is not only a tumor suppressor in certain cancers, including breast, and head and neck cancer $(20,21)$, but is also a candidate as a highly specific marker for lung SCCs. A recent study determined that the expression of PTEN is regulated by miR-205 (22).

In our study miR-375 was overexpressed in AC in accordance with a previous report which found that miR-375, in combination with miR-21, miR-486 and miR-200b, distinguishes lung AC patients from normal subjects (15). miR-375 was also elevated in AC compared with SCC in esophageal carcinoma (23). miR-375 is reported to target PDK1 leading to inactivation of the PI3K pathway (24). The PI3K pathway is more activated in SCC than in AC (1). The expression levels of miR-196b, miR-205 and miR-375 may explain the difference in the PI3K activation status between AC and SCC, although this requires further clarification.

LCC is further divided into LCNEC, basaloid carcinoma, and lymphoepithelioma-like carcinoma. The LCNEC samples in our study expressed higher levels of miR-196b and miR-205, but lower levels of miR-375, demonstrating SCC-like properties. However, other LCC samples, with the exception of LCNECs, were accurately classified into the AC group by the three miRNA markers identified in this study. This finding suggests that it inappropriate to classify LCNEC into LCC according to the miRNA marker method. LCNEC is actually known to be genetically and immunohistochemically more similar to SCLC than NSCLC $(25,26)$.

Conventional histological subtyping of NSCLC, including immunohistochemical staining, has limitations with respect to the accurate diagnosis of NSCLC (5). There are certain cases in which samples are not capable of being evaluated by immunohistochemistry due to their small biopsy size or loss of immunoreactivity. Therefore, we believe that measuring miRNA expression levels may be a complementary diagnostic technique that assists with the immunohistochemical profiling of cancer biopsies with unknown histology.

In conclusion, we demonstrated that hsa-miR-196b, hsa-miR-205 and hsa-miR-375 are highly valuable molecular markers for the classification of NSCLC histologic subtypes.

\section{Acknowledgements}

The authors would like to thank Ms. Mikiko Shibuya for her technical assistance. This study was supported in part by Grants-in-Aid for Scientific Research on Priority Areas from the Ministry of Education, Culture, Sports, Science and Technology of Japan to K.S. (grant no. 22590870) and J.H. (grant no. 23790920).

\section{References}

1. Herbst RS, Heymach JV and Lippman SM: Lung Cancer. N Engl J Med 359: 1367-1380, 2008.

2. Scagliotti GV, Parikh P, von Pawel J, et al: Phase III study comparing cisplatin plus pemetrexed in chemotherapy-naive patients with advanced-stage non-small-cell lung cancer. J Clin Oncol 26: 3543-3551, 2008.

3. Ciuleanu T, Brodowicz T, Zielinski C, et al: Maintenance pemetrexed plus best supportive care versus placebo plus best supportive care for non-small-cell lung cancer: a randomised, double-blind, phase 3 study. Lancet 374: 1432-1440, 2009.

4. Gridelli C, Ardizzoni A, Douillard JY, et al: Recent issues in first-line treatment of advanced non-small-cell lung cancer: Results of an International Expert Panel Meeting of the Italian Association of Thoracic Oncology. Lung Cancer 68: 319-331, 2010.

5. Rossi G, Pelosi G, Graziano P, Barbareschi M and Papotti M: A reevaluation of the clinical significance of histological subtyping of non-small-cell lung carcinoma: diagnostic algorithms in the era of personalized treatments. Int J Surg Pathol 17: 206-218, 2009.

6. Mok TS, Wu YL, Thongprasert S, et al. Gefitinib or carboplatinpaclitaxel in pulmonary adenocarcinoma. N Engl J Med 361: 947-957, 2009.

7. Kawada I, Soejima K, Watanabe H, et al: An alternative method for screening EGFR mutation using RFLP in non-small cell lung cancer patients. J Thorac Oncol 3: 1096-1103, 2008.

8. Sandler A, Gray R, Perry MC, et al: Paclitaxel-carboplatin alone or with bevacizumab for non-small-cell lung cancer. N Engl J Med 355: 2542-2550, 2006.

9. Esquela-Kerscher A and Slack FJ: Oncomirs - microRNAs with a role in cancer. Nat Rev Cancer 4: 259-269, 2006.

10. Raponi D, Dossey L, Jatkoe T, et al: MicroRNA classifiers for predicting prognosis of squamous cell lung cancer. Cancer Res 69: 5776-5783, 2009.

11. Yanaihara N, Caplen N, Bowman E, et al: Unique microRNA molecular profiles in lung cancer diagnosis and prognosis. Cancer Cell 3: 189-198, 2006

12. Colby TV, Noguchi M, Henschke C, et al: Adenocarcinoma. In: World Health Organization classification of tumours. Pathology and Genetics. Tumours of the Lung, Pleura, Thymus and Heart. Travis WD, Brambilla E, Muller-Hemelink HK and Harris CC (eds). IARC Press, Lyon, pp35-44, 2005.

13. Hammar SP, Brambilla C, Pugatch B, et al: Squamous cell carcinoma. In: World Health Organization classification of tumours. Pathology and Genetics. Tumours of the Lung, Pleura, Thymus and Heart. Travis WD, Brambilla E, Muller-Hemelink HK and Harris CC, (eds). IARC Press, Lyon, pp26-30, 2005.

14. Lebanony D, Benjamin H, Gilad S, et al: Diagnostic assay based on hsa-miR-205 expression distinguishes squamous from nonsquamous non-small-cell lung carcinoma. J Clin Oncol 27: 2030-2037, 2009.

15. Yu L, Todd NW, Xing L, et al: Early detection of lung adenocarcinoma in sputum by a panel of microRNA markers. Int J Cancer 127: 2870-2878, 2010.

16. Bishop JA, Benjamin H, Cholakh H, Chajut A, Clark DP and Westra WH: Accurate classification of non-small cell lung carcinoma using a novel microRNA-based approach. Clin Cancer Res 16: 610-619, 2010.

17. Wang YX, Zhang XY, Zhang BF, Yang CQ, Chen XM and Gao HJ: Initial study of microRNA expression profiles of colonic cancer without lymph node metastasis. J Dig Dis 11: 50-54, 2010.

18. Popovic R, Riesbeck LE, Velu CS, et al: Regulation of mir-196b by MLL and its overexpression by MLL fusions contributes to immortalization. Blood 113: 3314-3322, 2009. 
19. Schimanski CC, Berger M, Rahman F, et al: High miR-196a levels promote the oncogenic phenotype of colorectal cancer cells. World J Gastroenterol 15: 2089-2096, 2009.

20. Wu H and Mo YY: Targeting miR-205 in breast cancer. Expert Opin Ther Targets 13: 1439-1448, 2009.

21. Childs G, Fazzari M, Kung G, et al: Low-level expression of microRNAs let-7d and miR-205 are prognostic markers of head and neck squamous cell carcinoma. Am J Pathol 17: 736-745, 2009.

22. Greene SB, Gunaratne PH, Hammond SM, and Rosen JM: A putative role for microRNA-205 in mammary epithelial cell progenitors. J Cell Sci 123: 606-618, 2010.

23. Mathé EA, Nguyen GH, Bowman ED, et al: MicroRNA expression in squamous cell carcinoma and adenocarcinoma of the esophagus: associations with survival. Clin Cancer Res 15: 6192-6200, 2009.
24. El Ouaamari A, Baroukh N, Martens GA, Lebrun P, Pipeleers $\mathrm{D}$ and van Obberghen E: miR-375 targets 3'-phosphoinositidedependent protein kinase-1 and regulates glucose-induced biological responses in pancreatic beta-cells. Diabetes 57: 27082717, 2008 .

25. Saji H, Tsuboi M, Matsubayashi J, et al: Clinical response of large cell neuroendocrine carcinoma of the lung to perioperative adjuvant chemotherapy. Anticancer Drugs 21: 89-83, 2010.

26. Kozuki T, Fujimoto N, Ueoka H, et al: Complexity in the treatment of pulmonary large cell neuroendocrinecarcinoma. J Cancer Res Clin Oncol 131: 147-51, 2005. 